\title{
Electrochemical Detection of Oxacillin Resistance using Direct- Labelling Solid-Phase Isothermal Amplification
}

\section{Supporting Information}

\author{
Adrian Butterworth ${ }^{1}$, Pratibha Pratibha², Andreas Marx², Damion K. Corrigan ${ }^{1 *}$ \\ ${ }^{1}$ Department of Biomedical Engineering, Wolfson Centre, 106 Rottenrow East, University of Strathclyde, \\ Glasgow G1 1XQ, UK \\ ${ }^{2}$ Department of Chemistry, Konstanz Research School Chemical Biology, University of Konstanz, Universitätsstraße \\ 10, 78457 Konstanz, Germany \\ *Corresponding author: damion.corrigan@strath.ac.uk
}

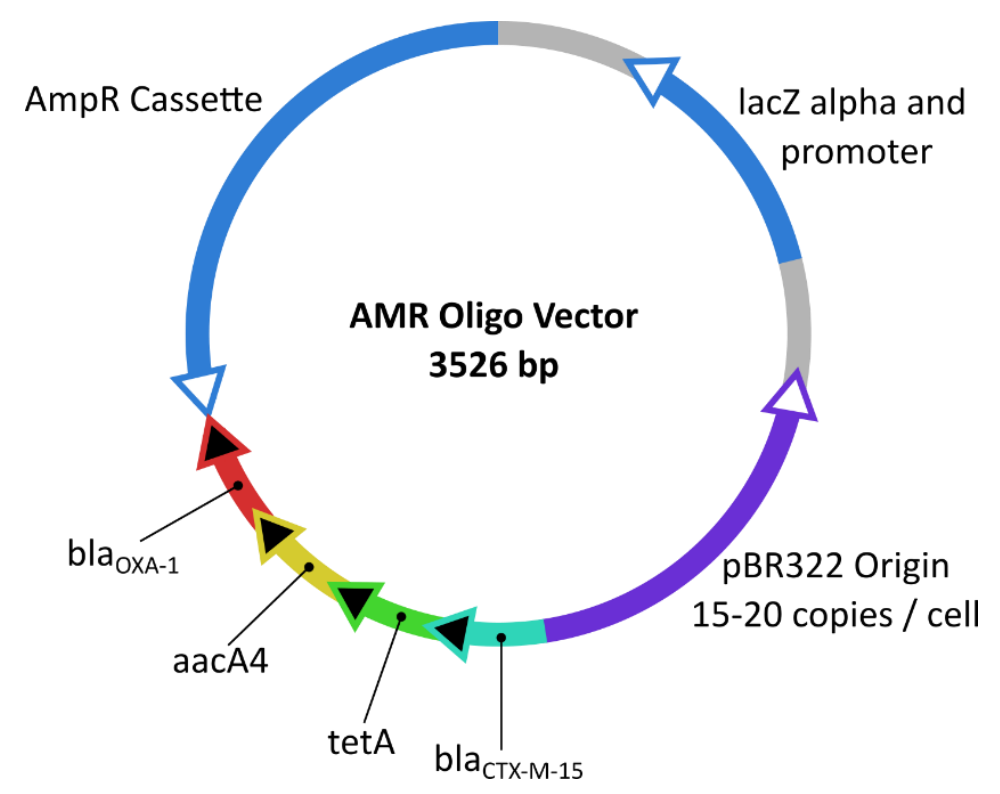

Figure S1. Diagram of AMR resistance plasmid designed for use in this work. 4 AMR gene segments were incorporated into the plasmid for future multiplexed testing. 
A

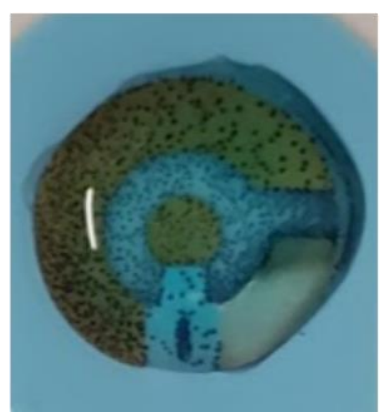

B

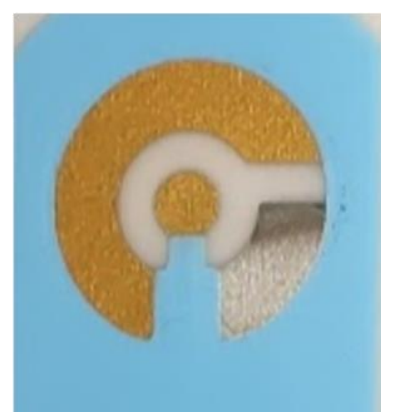

Figure S2. Nucleation sites for TMB formation and degradation of Silver reference electrode following 20-minutes incubation with $\mathrm{H}_{2} \mathrm{O}_{2}$ and $\mathrm{TMB}$

\section{AMR PLASMID SEQUENCE}

The AMR resistance plasmid sequence used is as follows (forward strand provided).

TGTATTTAGAAAAATAAACAAATAGGGGTTCCGCGCACATTTCCCCGAAAAGTGCCACCTGACGTCTAAGAAACCATTATTATCATGACATTA ACCTATAAAAATAGGCGTATCACGAGGCCCTTTCGTCTCGCGCGTTTCGGTGATGACGGTGAAAACCTCTGACACATGCAGCTCCCGGAGAC GGTCACAGCTTGTCTGTAAGCGGATGCCGGGAGCAGACAAGCCCGTCAGGGCGCGTCAGCGGGTGTTGGCGGGTGTCGGGGCTGGCTTAA CTATGCGGCATCAGAGCAGATTGTACTGAGAGTGCACCATATGCGGTGTGAAATACCGCACAGATGCGTAAGGAGAAAATACCGCATCAGG CGCCATTCGCCATTCAGGCTGCGCAACTGTTGGGAAGGGCGATCGGTGCGGGCCTCTTCGCTATTACGCCAGCTGGCGAAAGGGGGATGTG CTGCAAGGCGATTAAGTTGGGTAACGCCAGGGTTTTCCCAGTCACGACGTTGTAAAACGACGGCCAGTGAATTCGAGCTCGGTACCCGGGG ATCCTCTAGAGTCGACCTGCAGGCATGCAAGCTTGGCGTAATCATGGTCATAGCTGTTTCCTGTGTGAAATTGTTATCCGCTCACAATTCCACA CAACATACGAGCCGGAAGCATAAAGTGTAAAGCCTGGGGTGCCTAATGAGTGAGCTAACTCACATTAATTGCGTTGCGCCGCTCGAAGAGCA CCCAGTCGGGAAACCTGTCGTGCCAGCTGCATTAATGAATCGGCCAACGCGCGGGGAGAGGCGGTTTGCGTATTGGGCGCTCTTCCGCTTCC TCGCTCACTGACTCGCTGCGCTCGGTCGTTCGGCTGCGGCGAGCGGTATCAGCTCACTCAAAGGCGGTAATACGGTTATCCACAGAATCAGG GGATAACGCAGGAAAGAACATGTGAGCAAAAGGCCAGCAAAAGGCCAGGAACCGTAAAAAGGCCGCGTTGCTGGCGTTTTTCCATAGGCTC CGCCCCCCTGACGAGCATCACAAAAATCGACGCTCAAGTCAGAGGTGGCGAAACCCGACAGGACTATAAAGATACCAGGCGTTTCCCCCTGG AAGCTCCCTCGTGCGCTCTCCTGTTCCGACCCTGCCGCTTACCGGATACCTGTCCGCCTTTCTCCCTTCGGGAAGCGTGGCGCTTTCTCATAGCT CACGCTGTAGGTATCTCAGTTCGGTGTAGGTCGTTCGCTCCAAGCTGGGCTGTGTGCACGAACCCCCCGTTCAGCCCGACCGCTGCGCCTTAT CCGGTAACTATCGTCTTGAGTCCAACCCGGTAAGACACGACTTATCGCCACTGGCAGCAGCCACTGGTAACAGGATTAGCAGAGCGAGGTAT GTAGGCGGTGCTACAGAGTTCTTGAAGTGGTGGCCTAACTACGGCTACACTAGAAGGACAGTATTTGGTATCTGCGCTCTGCTGAAGCCAGT TACCTTCGGAAAAAGAGTTGGTAGCTCTTGATCCGGCAAACAAACCACCGCTGGTAGCGGTGGTTTTTTTGTTTGCAAGCAGCAGATTACGCG CAGAAAAAAAGGATCTCAAGAAGATCCTTTGATCTTTTCTACGGGGTCTGACCTCGCTGCACCGGTGGTATTGCCTTTCATCCATGTCACCAGC TGCGCCCGTTGGCTGTCGCCCAATGCTTTACCCAGCGTCAGATTCCGCAGAGTTTGCGCCATTGCCCGAGGTGAAGTGGTATCACGCGGATCG CCCGGAATGGCGGTGTTTAACGTCGGCTCGGTACGGTCGAGACGGAACGTTTCGTCTCCCAGCTGGGGGTCTGACGACCCAAAGCGCGGCC GGCACCTGTCCGACAAGTTGCATGATGAAGAAGACCGCCATCAGGGCGGCGACGACGGTCATGCCCCGGGCCCACCGGAACGAAGCGAGC GGGTTGAGAGCCTCCCGGCGTAACGGCCGGCGTTCGCCTTTGTGCGACTCCGGCAAAAGGAAACAGCCCGTCAGGAAATTGAGGCCGTTCA AGGGGGGTCTGACCAACAGTGCCTTTAAGCGTGCATAATAAGCCCTACACAAATTGGGAGTTAGACATCATGAGCAACGCAAAAACAAAGTT AGGCATCACAAAGTACAGCATCGTGACCAACAGCAACGATTCCGTCACACTGCGCCTCATGACTGAGCATGACCTTGCGATGCTCTATGAGTG GCTAAATCGATCTCATATCGTCGAGTGGGGGGTCTGACCTCAAAGATTTTGATTATGGAAATCAAGACTTCTCTGGAGATAAAGAAAGAAAC AACGGATTAACAGAAGCATGGCTCGAAAGTAGCTTAAAAATTTCACCAGAAGAACAAATTCAATTCCTGCGTAAAATTATTAATCACAATCTC CCAGTTAAAAACTCAGCCATAGAAAACACCATAGAGAACATGTATCTACAAGAGGGGTCTGACGCTCAGTGGAACGAAAACTCACGTTAAGG GATTTTGGTCATGAGATTATCAAAAAGGATCTTCACCTAGATCCTTTTAAATTAAAAATGAAGTTTTAAATCAATCTAAAGTATATATGAGTAA ACTTGGTCTGACAGTTACCAATGCTTAATCAGTGAGGCACCTATCTCAGCGATCTGTCTATTTCGTTCATCCATAGTTGCCTGACTCCCCGTCGT GTAGATAACTACGATACGGGAGGGCTTACCATCTGGCCCCAGTGCTGCAATGATACCGCGAGAACCACGCTCACCGGCTCCAGATTTATCAG CAATAAACCAGCCAGCCGGAAGGGCCGAGCGCAGAAGTGGTCCTGCAACTTTATCCGCCTCCATCCAGTCTATTAATTGTTGCCGGGAAGCT AGAGTAAGTAGTTCGCCAGTTAATAGTTTGCGCAACGTTGTTGCCATTGCTACAGGCATCGTGGTGTCACGCTCGTCGTTTGGTATGGCTTCA TTCAGCTCCGGTTCCCAACGATCAAGGCGAGTTACATGATCCCCCATGTTGTGCAAAAAAGCGGTTAGCTCCTTCGGTCCTCCGATCGTTGTCA GAAGTAAGTTGGCCGCAGTGTTATCACTCATGGTTATGGCAGCACTGCATAATTCTCTTACTGTCATGCCATCCGTAAGATGCTTTTCTGTGAC TGGTGAGTACTCAACCAAGTCATTCTGAGAATAGTGTATGCGGCGACCGAGTTGCTCTTGCCCGGCGTCAATACGGGATAATACCGCGCCAC ATAGCAGAACTTTAAAAGTGCTCATCATTGGAAAACGTTCTTCGGGGCGAAAACTCTCAAGGATCTTACCGCTGTTGAGATCCAGTTCGATGT AACCCACTCGTGCACCCAACTGATCTTCAGCATCTTTTACTTTCACCAGCGTTTCTGGGTGAGCAAAAACAGGAAGGCAAAATGCCGCAAAAA AGGGAATAAGGGCGACACGGAAATGTTGAATACTCATACTCTTCCTTTTTCAATATTATTGAAGCATTTATCAGGGTTATTGTCTCATGAGCGG ATACATATTTGAA 